\section{Highlights from Recent Literature}

\section{Analytical}

\subsection{Direct Voltammetric Determination of Gold Nanoparticles Using Graphite-Epoxy Composite Electrode}

A new voltammetric method for a direct detection of Gold nanoparticles, based on adsorption and electrochemical detection of colloidal Gold, is described. M. Pumera, M. Aldavert, C. Mills, A. Merkoci and S. Alegret from the Departament de Quimica, Grup de Sensors i Biosensors, Universitat Goldtonoma de Barcelona, Spain, Electrochimica Acta 2005, 50(18), 3702. In this protocol, the absorption of Gold nanoparticles onto the rough surface of graphite-epoxy composite electrode is followed by their electrochemical oxidation in $0.1 \mathrm{M} \mathrm{HCl}$ medium at a potential of $+1.25 \mathrm{~V}$. The resulting tetrachloroaurate ions generated near the electrode surface are detected by differential pulse voltammetry (DPV). The surface characteristics of the composite electrode were studied and the parameters that affect the complete analytical detection process of Gold nanoparticles are optimized.

\subsection{Use of ICP and XAS to Determine the Enhancement of Gold Phytoextraction by Chilopsis Linearis using Thiocyanate as a Complexing Agent}

Under natural conditions gold has low soly. that decreases its bioavailability, a critical factor for phytoextraction. Phytoextraction can be improved by using synthetic chelating agents. Preliminary studies have shown that the desert willow (Chilopsis linearis) is able to extract gold from a gold-enriched medium. J. Gardea-Torresdey, E. Rodriguez, J. Parsons, J. Peralta-Videa, G. Meitzner, G. Cruz-Jimenez, from the Environmental Science and Engineering PhD Program, University of Texas at El Paso, USA, Analytical and Bioanalytical Chemistry 2005, 382(2), 347 The objective was to detect the ability of thiocyanate to enhance the gold-uptake capacity of C. linearis. Seedlings of this plant were exposed to various hydroponics treatment involving 2.5x 10-5 M Gold solution and 0-10-4 $\mathrm{M} \mathrm{NH}_{4} \mathrm{SCN}$. Each treatment had its respective control. After two weeks the effect of the treatment on plant growth and gold content was detected by using inductively coupled plasma (ICP) optical emission spectroscopy. No signs of shootgrowth inhibition were observed at any $\mathrm{NH}_{4} \mathrm{SCN}$ treatment level used. The addition of $10-4 \mathrm{~mol} / \mathrm{L} \mathrm{NH}_{4} \mathrm{SCN}$ increased the concentration of gold by $\approx 595,396$, and $467 \%$ in the roots, stems, and leaves, respectively $\mathrm{X}$-ray absorption spectroscopy (XAS) studies showed that the oxidation state of gold was $\mathrm{Au}(0)$ and that gold nanoparticles were formed inside the plants.

\section{Catalysis}

\subsection{Electrocatalytic Oxidation of Glucose at Gold Nanoparticle-Modified Carbon Electrodes in Alkaline And Neutral Solutions}

Electrocatalytic oxidation of glucose in alkaline and neutral solutions was examined using carbon electrodes modified with $2 \mathrm{~nm}$ core sized gold nanoparticles (Gold2 nm nanoparticle). M. Tominaga, T. Shimazoe, M. Nagashima, I. Taniguchi from the Department of Applied Chemistry and Biochemistry, Faculty of Engineering, Kumamoto University, Kumamoto, 860-8555, Japan SO Electrochemistry Communications (2005), 7(2), 189. The voltammetric responses for the electrocatalytic oxidation of glucose at Au2 nm nanoparticle-modified electrodes in both alkaline and neutral solutions were similar to those at a gold plate electrode. However, the different results were obtained for the electrolysis of glucose between Au2 nm nanoparticle-modified and gold plate electrodes. The current flow due to the oxidation of glucose during electrolysis at the gold plate electrode decreased quickly with time, due to the electrode surface poisoning. On the other hand, the decrease in current flow at the Au2 $\mathrm{nm}$ nanoparticlemodified electrode was much improved in both alkaline and neutral solutions The electrolytic oxidation of glucose was performed at Au2 nm nanoparticle modified electrodes in both alkaline and neutral solutions. In the alkaline solution, at a potential of $-0.3 \mathrm{~V}$, gluconolactone (or gluconate) was formed with a current efficiency of $100 \%$, while at potentials of -0.1 to $0.3 \mathrm{~V}$, oxalate and gluconolactone (or gluconate) were obtained as main products. In a neutral solution, electrolysis at 0.1 and $0.3 \mathrm{~V}$, gluconolactone (or gluconate) was obtained with current efficiencies of $88-100 \%$.

\subsection{Effect of Supporting Surface Layers on Catalytic Activities of Gold Nanoparticles in CO Oxidation}

The surfaces of fumed silica materials were modified with a surface sol-gel process for catalysis applications. W. Yan, S. Mahurin, B. Chen, S. Overbury, S. Dai from the Chemical Sciences Division, Oak Ridge National Laboratory, Oak Ridge, USA, Journal of Physical Chemistry B 2005, 109(32), 15489

This surface-modification approach allows not only a monolayer growth of $\mathrm{TiO}_{2}$ or $\mathrm{Al}_{2} \mathrm{O}_{3}$ but also a stepwise double-layer growth of $\mathrm{TiO}_{2} / \mathrm{TiO}_{2}, \mathrm{Al}_{2} \mathrm{O}_{3} / \mathrm{Al}_{2} \mathrm{O}_{3}, \mathrm{TiO}_{2} / \mathrm{Al}_{2} \mathrm{O}_{3}$, or $\mathrm{Al}_{2} \mathrm{O}_{3} / \mathrm{TiO}_{2}$ on the surfaces of the silica materials with a monolayer precision. XRD analyses revealed that the coated monolayers and double layers of $\mathrm{TiO}_{2}$ and $\mathrm{Al}_{2} \mathrm{O}_{3}$ were amorphous. Gold nanoparticles were successfully deposited on the above six surface-modified silica materials via a deposition-precipitation method. The catalytic activities of these six gold catalysts for $\mathrm{CO}$ oxidation are highly dependent on the structures of their surface monolayers or double layers. The gold catalyst supported on the silica material functionalized with a $\mathrm{TiO}_{2}$ monolayer $\left(\mathrm{Au} / \mathrm{TiO}_{2}\right)$ is the most active in both as-synthesized and oxidized forms, while 
the gold catalyst supported on the silica material functionalized with an $\mathrm{Al}_{2} \mathrm{O}_{3} / \mathrm{TiO}_{2}$ double layer $\left(\mathrm{Au} / \mathrm{Al}_{2} \mathrm{O}_{3} / \mathrm{TiO}_{2} / \mathrm{SiO}_{2}\right)$ is the most active in the reduced form among the six catalysts. Surprisingly, the gold catalyst supported on the silica material functionalized with a $\mathrm{TiO}_{2} / \mathrm{Al}_{2} \mathrm{O}_{3}$ double layer $\left(\mathrm{Au} / \mathrm{TiO}_{2} / \mathrm{Al}_{2} \mathrm{O}_{3} / \mathrm{SiO}_{2}\right)$ has much less activity than $\mathrm{Au} / \mathrm{Al}_{2} \mathrm{O}_{3} / \mathrm{TiO}_{2} / \mathrm{SiO}_{2}$ under all various treatments, underscoring the sensitivity of the catalytic activity to the structure of the supporting surfaces.

\subsection{Low Temperature and Low Pressure CO Oxidation on Gold Clusters Supported on Mgo(100)}

$\mathrm{CO}$ oxidation has been investigated on $\mathrm{Au} / \mathrm{MgO}(100)$ model catalysts at RT and low pressure. O. Meerson, G. Sitja, C. Henry from the CRMCN-CNRS, Marseille, European Physical Journal D: Atomic, Molecular and Optical Physics 2005, 34(1-3), 119. The gold particles prepared by UHV evaporation on clean $\mathrm{MgO}$ surfaces are characterized by HRTEM. The gold particles are FCC single crystals or multiple twins with five-fold symmetry. IR spectroscopy indicates that two types of adsorption sites are present which correspond to loosely and strongly bound $\mathrm{CO}$. The equil. CO coverage for the strongly bound $\mathrm{CO}$ is smaller than $0.1 \mathrm{ML}$. CO titration experiments show that oxygen does not dissociate on the gold nanoparticles. The CO oxidation reaction is studied at RT by mol. beam methods. A steady state $\mathrm{CO}$ reaction probability up to 0.50 is measured, for the first time at low pressure, for gold particles with a mean size of $1.5 \mathrm{~nm}$. A reaction mechanism is proposed in which $\mathrm{CO}$ adsorbed on low coordinated gold atoms reacts with oxygen adsorbed molecularly, possibly at the Au/MgO interface.

\subsection{Co Oxidation Catalyzed by Supported Gold: Cooperation between Gold and Nanocrystalline Rare-Earth Supports Forms Reactive Surface Superoxide and Peroxide Species}

Beneficial defects: Reactive .eta.1-superoxide and peroxide species at one-electron defect sites are formed at the metalsupport interface in gold nanoclusters on nanocryst. $\mathrm{CeO}_{2}-\mathrm{X}$. J Guzman, S Carrettin, J Fierro-Gonzalez, Y Hao, B Gates, A Corma from the Instituto de Tecnologia Quimica UPV-CSIC Universidad Politecnica de Valencia,Spain, Angewandte Chemie, International Edition 2005, 44(30), 4778- These intermediates oxidize $\mathrm{CO}$ adsorbed on gold to form $\mathrm{CO}_{2}$. The reactive oxygen species are formed on nanocryst. $\mathrm{CeO}_{2}$ $x$ but not on conventional $\mathrm{CeO}_{2}$, and their formation is enhanced by the gold.

\subsection{Gold Supported $\mathrm{CeO}_{2} / \mathrm{Al}_{2} \mathrm{O}_{3}$ Catalysts for $\mathrm{CO}$ Oxidation: Influence of the Ceria Phase}

A series of low loading gold supported ceria/alumina catalysts have been prepared by the deposition-precipitation method, varying the $\mathrm{pH}$ of the synthesis. The catalysts were characterized by means of XRD, TEM, SBET, XRF and UV-Vis techniques, and their catalytic activity towards $\mathrm{CO}$ oxidation in the absence and in presence of water in the stream, were tested. M. Centeno, C. Portales, I. Carrizosa, J. Odriozola from the Institute de Ciencia de Materiales de Sevilla, Centro Mixto, Spain, Catalysis Letters 2005, 102(3-4), 289. It has been found that in this low loading gold catalysts, where the metallic particles are far away one from another and the oxygen transportation is not the limiting step of the reaction, the electronic properties of the ceria phase and the structure of the metal-support perimeter more than the diameter of the gold nanoparticles is the determinant factor in the catalytic performances of the solid.

\subsection{Catalytic Oxidation of 1,2-diols to $\alpha$-hydroxy- carboxylates with stabilized Gold Nanocolloids combined with a Membrane- based Catalyst Separation}

Gold (Au) nanosols stabilized with poly(vinyl alcohol) can be used as homogeneous oxidation catalysts, both in water and in a variety of alcohol solvents. Under 0.5 $\mathrm{MPa} \mathrm{O}_{2}$ pressure, 1,2-diols with varying chain length, such as 1,2-propanediol or 1,2-octanediol, are oxidized to the corresponding $\alpha$-hydroxy carboxylic acids with total chemoselectivity. P. Mertens, M. Bulut, L. Gevers, I. Vankelecom, P. Jacobs, D. De Vos from the Centre for Surface Chemistry and Catalysis, Belgium, Catalysis Letters 2005, 102(1-2), 57 . The activity of the sol is of the same order as previously reported for stabilized nanoparticles deposited on carbon supports. The stabilized sols retain their activity over extended periods. The gold metal colloids can efficiently be recycled by means of a nanofiltration, either from an aqueous reaction medium, or from an org. solvent. The most suitable membranes for the gold sol recuperation are cellulose acetate membranes for the aqueous filtration, and poly(dimethyl)siloxane membranes for the solvent filtration. Recycling tests after nanofiltration show that catalytic activity is largely preserved in consecutive runs.

\subsection{Application of Gold Catalysis In the Synthesis of Heterocyclic Systems}

A review by $A$. Arcadi, G. Bianchi from the Dipartimento di Chimica, Ingegneria Chimica e Materiali, Facolta di Scienze, Universita di L'Aquila, Coppito, Italy, Targets in Heterocyclic Systems 2004, 8, 82. Gold catalysis accomplishes the functionalization of a variety of heterocyclic derivatives through Gold-catalyzed C-C bond formation reactions involving $\mathrm{C}-\mathrm{H}$ bond cleavage. Gold activation of alkynes towards intramol. nucleophilic attack represents a powerful tool for annulation processes. The many personalities of Gold as Lewis acid, transition-metal and dual role catalyst allow innovative synthetic approaches through domino processes. 


\subsection{Studies on Gold Nanoparticles supported on Iron, Cobalt, Manganese, and Cerium Oxide Catalytic Materials}

Gold nanoparticles containing iron, cobalt, manganese, and cerium materials were prepared using precipitationdeposition/co-precipitation methods. D. Akolekar, S. Bhargava, G. Foran, M. Takahashi from the Catalysis and Advanced Materials Research Group, Department of Applied Chemistry, RMIT University, Melbourne, Australia, Journal of Molecular Catalysis A: Chemical 2005, 238, 1 The techniques employed for the characterization of these materials were ICP-MS, TCA, TEM, XRD, BET, Moessbauer, and XAS. Au L3-edge $X$-ray absorption spectroscopic measurements were carried out over a series of transition and rare earth materials containing gold nanoparticles. The size of gold nanoparticles varied in the range of $\approx 3$ to $7 \mathrm{~nm}$ in the $\mathrm{Fe} / \mathrm{Co} / \mathrm{Mn} / \mathrm{Ce}$ materials. These materials possess medium between 34 and $63 \mathrm{~m} 2 / \mathrm{g}$. The XRD analysis of the samples indicated the high phase purity and crystallinity of the support $(\mathrm{Fe} / \mathrm{Co} / \mathrm{Mn} / \mathrm{Ce})$ materials. Moessbauer spectroscopy was employed to obtain valuable information on the electronic and crystallographic structure of gold nanoparticles. An X-ray absorption fine structure (XANES, EXAFS) technique was used in obtaining critical information about the atomic distances, bonding, and neighbouring environment for gold atoms in the $\mathrm{Fe} / \mathrm{Co} / \mathrm{Mn} / \mathrm{Ce}$ materials. XAFS and in situ high temperature XRD techniques were used to investigate the influence of the high temperature treatment on gold nanoparticles inside the metal oxide materials. The results obtained from XAFS, XANES, XRD, and Moessbauer analysis confirm the typical characteristics and structure of gold nanoparticles in these materials.

\subsection{The Nature of the Oxidation States of Gold on $\mathrm{ZnO}$}

The interaction between gold in the 0, I, II and III oxidation states and the zinc-terminated $\mathrm{ZnO}(0001)$ surface is studied via the QM/MM electronic embedding method using d. functional theory. The surface sites considered are the vacant zinc interstitial surface site (VZISS) and the bulkterminated island site (BTIS). N. Phala, G. Klatt, E. van Steen, S. French, A. Sokol, C. Catlow from the Catalysis Research Unit, Department of Chemical Engineering, University of Cape Town, South Africa, Physical Chemistry Chemical Physics, 2005, 7(12), 2440, find that on the VZISS, only Au(0) and $A u(i)$ are stable oxidation states. However, all clusters of I to III oxidation states are stable as substitutionals for $\mathrm{Zn}^{2+}$ in the bulk terminated island site. $\mathrm{Au}(\mathrm{OH}) \mathrm{x}$ complexes $(\mathrm{x}=1-3)$ can adsorb exothermically onto the VZISS, indicating that higher oxidation states of gold can be stabilized at this site in the presence of hydroxyl groups. $\mathrm{CO}$ is used as a probe mol. to study the reactivity of Gold in different oxidation states in VZISS and BTIS. In all cases, the authors find that the strongest binding of $\mathrm{CO}$ is to surface $\mathrm{Au}(\mathrm{i})$. Furthermore, $\mathrm{CO}$ binding onto $A u(0)$ is stronger when the gold atom is adsorbed onto the VZISS compared to CO binding onto a gas phase neutral gold atom. These results indicate that the nature of the oxidation states of Gold on $\mathrm{ZnO}(0001)$ will depend on the type of adsorption site. The role of $\mathrm{ZnO}$ in $\mathrm{Au} / \mathrm{ZnO}$ catalysts is not, therefore, merely to disperse gold atoms/particles, but to also modify their electronic properties.

\subsection{Platinum and Gold Nanoparticles Generated in Spherical Polyelectrolyte Brushes and their Catalytic Activity}

A novel catalyst system was developed that worked in aqueous solutions under mild conditions. M. Ballauff, G. Sharma, R. Kempe, T. Irrgang, Y. Talmon, S. Proch from the Physikalische Chemie, University of Bayreuth, Bayreuth, Germany, Polymer Preprints (American Chemical Society, Division of Polymer Chemistry) 2005, 46(2), 56. The immobilization of Pt- or Gold-nanoparticles within cationic spherical polyelectrolytes was performed, and these catalysts were used for the heterogeneous hydrogenation of butyraldehyde. The metals were catalytically active and suitable for several cycles.

\subsection{Gold Catalyst, Production Method and Oxidation of $\mathrm{CO}$}

The invention refers to a gold catalyst for the oxidation of $\mathrm{CO}$ formed by dispersing Gold particles in an aqueous surfactant solution using a UV or visible laser, and adding $\psi$-alumina particles so that the gold particles are supported on the alumina, then sepg. and washing the gold-supporting alumina. J. Kono, T. Sakai, Y. Takeda, F. Mafune, T. Kondo from the Toyota Motor Corp., Japan; Genesis Research Institute Inc., Patent No., JP 2005230699, A2, 2005.

\subsection{Activation of $\mathrm{CO}, \mathrm{O}_{2}$ and $\mathrm{H}_{2}$ on Gold-Based Catalysts}

$\mathrm{Au} / \mathrm{CeOx} / \mathrm{Al}_{2} \mathrm{O}_{3}$ is highly active for $\mathrm{CO}$ oxidation at low temps. and full conversion is already achieved around $60^{\circ} \mathrm{C}$. From experimental results, it is concluded that ceria can act as oxygen supplier probably via Mars and van Krevelen mechanism. A. Cluhoi, H. Vreeburg, J. Bakker, B. Nieuwenhuys from the Department of Heterogeneous Catalysis and Surface Chemistry, Leiden Institute of Chemistry, Leiden University, Netherlands, Applied Catalysis, A: General 2005, 291 1. The $\mathrm{H}_{2}-\mathrm{D}_{2}$ isotopic exchange reaction is also efficiently catalyzed by $\mathrm{Au} / \mathrm{Al}_{2} \mathrm{O}_{3}$. The gold particle size is of crucial importance for high activity. Most likely, a gold surface rich in defects activates the $\mathrm{H}_{2}\left(D_{2}\right)$ mols.

\subsection{Low Temperature CO Oxidation on Supported and Unsupported Gold Compounds}

Several simple gold compounds and their phys. mixtures with $\mathrm{TiO}_{2}$ were tested for low temperature $\mathrm{CO}$ oxidation J. Soares, M. Bowker from the School of Chemistry, University of Reading, UK, Applied Catalysis, A: General 2005, 291, 1. No true catalytic activity was found for gold precursors on their own, although both $\mathrm{Au}_{2} \mathrm{O}_{3}$ and $\mathrm{Au}(\mathrm{OH})_{3}$ react well with 
CO even at room temperature in a non-catalytic manner. Despite that catalytic activity was obtained by phys. mixing $\mathrm{Au}(\mathrm{OH})_{3}$ or $\mathrm{Au}_{2} \mathrm{O}_{3}$ with $\mathrm{TiO}_{2}$ and the results further emphasize the importance of a good contact between the gold and the support for good CO oxidation activity.

\subsection{Characterization of Nanosized Gold, Silver and Copper Catalysts Supported on Ceria}

A comparative study of $\mathrm{CO}$ adsorption on nanosized $\mathrm{Au}, \mathrm{Ag}$ and Cu supported on ceria has been performed. T. Tabakova, F. Boccuzzi, M. Manzoli, A. Chiorino, D. Andreeva from the Institute of Catalysis, Bulgarian Academy of Sciences, Sofia, Bulgaria, Studies in Surface Science and Catalysis 2005, 155(Oxide Based Materials), 493. HRTEM images combined with EDS anal. have shown presence of highly dispersed metallic particles on the surface of the catalysts. FTIR results indicate that IB metals cause a strong modification of the surface properties of ceria, which leads to the appearance of more co-ordinatively unsatd. sites on the surface. Reduced $\mathrm{Au} / \mathrm{CeO}_{2}$ activates $\mathrm{CO}$ mols. on neg. charged nano-gold particles near to the oxygen vacancies on the ceria surface. FTIR spectra of CO adsorbed on oxidized Cu/ceria show an easy generation of metallic copper even at $90 \mathrm{~K}$. Ag/ceria catalyst is almost completely unable to adsorb and activate CO mols. due to the low adsorption energy for CO on silver.

\subsection{Nanostructure and Catalytic Properties of Gold Supported on Group IV Oxides}

The effect of the support on the HRTEM features and on the FTIR spectra of adsorbed $\mathrm{CO}$ at 90 and $300 \mathrm{~K}$ of $\mathrm{Au} / \mathrm{TiO}_{2}$, $\mathrm{Au} / \mathrm{ZrO}_{2}$ and $\mathrm{Au} / \mathrm{CeO}_{2}$, both oxidized and reduced; has been examined M. Manzoli, A. Chiorino, F. Boccuzzi from the Department of Chemistry I.F.M., University of Turin, Turin, Italy, Studies in Surface Science and Catalysis 2005, 155(Oxide Based Materials), 405 All catalysts have been prepared by the deposition-precipitation method. The aim of this work is to obtain information on the surface structure of the three catalysts in order to explain the differences observed in the catalytic activity in the CO oxidation reaction. The size and the size distribution of the metal particles produced on $\mathrm{Au} / \mathrm{TiO}_{2}$ and on $\mathrm{Au} / \mathrm{ZrO}_{2}$ are almost the same, $\mathrm{d}$.approximately equal to $4 \mathrm{~nm}$. On the contrary, the ceria supported sample exhibits Au particles that are significantly smaller, $\mathrm{d}$.approximately equal to $1 \mathrm{~nm}$, that are difficultly detected by HRTEM. FTIR data of CO adsorbed at $90 \mathrm{~K}$ on the three samples, either oxidized or reduced, and the spectroscopic evolution by heating up to RT, are presented and correlated with the different catalytic performances. In addition, data concerning the $\mathrm{CO}-\mathrm{O}_{2}$ interaction at different temps. will be discussed. The size of gold nanoparticles together with the size and the chem. nature of the oxidic supports are shown to be relevant in detg. firstly their durability and then the chemisorption and catalytic properties.

\subsection{Composition Based on Gold and Cerium Oxide, Preparation Process, and use as Catalyst, in Particular for the Oxidation of Carbon Monoxide}

The invention relates to a catalyst for the oxidation of carbon monoxide, composed of gold on a cerium oxide support, which is characterized in that its halogen content expressed by the molar ratio of halogen/gold is at the max. 0.04 and in that gold is present in the form of particles at most $10 \mathrm{~nm}$ in size. F. Fajardie, S. Verdier, K. Yokota from Rhodia Acetow G.m.b.H., Germany , Patent No FR 2866249, A1, August 2005 It is obtained by a process in which: a composition based on cerium oxide is placed in contact with a composition based on a gold halide by forming a suspension of these compounds; the $\mathrm{pH}$ of the medium thus formed is fixed at a value of at least 8 ; then the solid is separated from the reaction medium and the solid is washed with a basic solution. This composition can be used as a catalyst in processes involving oxidation of carbon monoxide: treatment of cigarette smoke, air purification, water gas shift reaction and preferential oxidation (PROX) in reforming.

\subsection{CO Oxidation on Gold Nanoparticles: Theoretical Studies}

I. Remediakis, N. Lopez, J. Norskov from the Center for Atomic-scale Materials Physics, Department of Physics, Technical University of Denmark, Applied Catalysis, A: General 2005, 291 1, present a summary of their theoretical results regarding CO oxidation on both oxide-supported and isolated gold nanoparticles. Using D. Functional Theory they have studied the adsorption of molecules and the oxidation reaction of $\mathrm{CO}$ on gold clusters. Low-coordinated sites on the gold nanoparticles can adsorb small inorganic molecules such as $\mathrm{O}_{2}$ and $\mathrm{CO}$, and the presence of these sites is the key factor for the catalytic properties of supported gold nanoclusters. Other contributions, induced by the presence of the support, can provide parallel channels for the reaction and modulate the final efficiency of Au-based catalysts. Finally, their theor. simulations allow them to discuss the selectivity of supported Gold nanoparticles.

\section{$2.18 \mathrm{CeO}_{2}$ Nanorods and Gold Nanocrystals Supported on $\mathrm{CeO}_{2}$ Nanorods as Catalyst}

The formation mechanism of uniform $\mathrm{CeO}_{2}$ structure at the nanometer scale via a wet-chem. reaction is of great interest in fundamental study as well as a variety of applications. In this work, large-scale well-crystallised $\mathrm{CeO}_{2}$ nanorods with uniform diameters in the range of 20-30 nm and lengths up to tens of micrometers are first synthesized through a hydrothermal synthetic route in $5 \mathrm{M} \mathrm{KOH}$ solution at $180{ }^{\circ} \mathrm{C}$ for $45 \mathrm{~h}$ without any templates and surfactants. P. Huang, F. Wu, B. Zhu, X. Gao, H. Zhu, T. Yan, W. Huang, S. Wu, $D$. Song from the Institute of New Energy Material Chemistry Department of Materials Chemistry, Nankai University, Tianjin, Peoples Republic of China, Journal of Physical Chemistry B 2005, 109(41), 19169. The nanorod formation involves 
dehydration of $\mathrm{CeO}_{2}$ nanoparticles and orientation growth along the [110] direction in $\mathrm{KOH}$ solution Subsequently, gold nanoparticles with crystallite sizes between 10 and $20 \mathrm{~nm}$ are loaded on the surface of $\mathrm{CeO}_{2}$ nanorods using $\mathrm{HAuCl}_{4}$ solution as the gold source and $\mathrm{NaBH}_{4}$ solution as a reducing agent. The synthesized $\mathrm{Au} / \mathrm{CeO}_{2}$ nanorods demonstrate a higher catalytic activity in $\mathrm{CO}$ oxidation than the pure $\mathrm{CeO}_{2}$ nanorods.

\subsection{The Unique Catalysis of Gold Nanoparticles}

A review by M. Haruta, Masatake from the National Institute of Advance Industrial Science and Technology, Tsukuba, Japan, Kagaku Kogyo 2004, 55(12), 905. The author reviews the unique catalytic properties of gold nanoparticles, including in the discussion the preparation of gold nanoparticle catalyst, the conditions under which the catalyst is activated, the effect of various catalyst supports, and the effect of structure on reaction mechanisms. The oxidation of $\mathrm{CO}$ is given as an example.

\subsection{Hydrogenation of Benzene Using Aqueous Solution of Polyoxometalates Reduced by CO over Gold Catalysts}

Aqueous polyoxometalate (H3PMo12O40) solution reduced by $\mathrm{CO}$ with liq. $\mathrm{H}_{2} \mathrm{O}$ using Gold nanoparticle catalysts at room temperature, which contains protons in liq. $\mathrm{H}_{2} \mathrm{O}$ and electrons associated with the reduced polyoxometalate, can produce gaseous $\mathrm{H}_{2}$ or can hydrogenate benzene over an electrochemical cell consisting of a simple $\mathrm{C}$ anode, $\mathrm{a}$ proton-exchange membrane, and a Pt- or Rh-based cathode. G. Rodriguez-Rivera, W. Kim, S. Evans, T. Voitl, J. Dumesic from the Department of Chemical and Biological Engineering, University of Wisconsin, Madison, USA, Journal of the American Chemical Society 2005, 127(31), 10790 In the present cell, $\mathrm{H}_{2}$ can be produced from the reduced H3PMo12O40 solution at voltages that are lower by $\approx 1.15 \mathrm{~V}$ compared to $\mathrm{H}_{2} \mathrm{O}$ electrolysis.

\section{Chemistry}

\subsection{Effects of Particle Size and Spacing on the Optical Properties of Gold Nanocrystals in Alumina}

Gold-particle $/ \mathrm{Al}_{2} \mathrm{O}_{3}$-matrix nanocomposite thin films with a narrow Gold particle size distribution were fabricated by radio-frequency magnetron cosputtering. J. Wang, W. Lau, Q. Li, from the Department of Physics, The Chinese University of Hong Kong, Hong Kong, Peop. Rep. China, Journal of Applied Physics 2005, 97(11), 114303/1. The films were characterized both chem. and structurally using XPS, x-ray diffraction, and transmission electron microscopy. The optical absorption of samples with various Gold particle sizes and concentrations were measured using an UV/visiblespectrometer. Both the Gold nanoparticle size and the Gold concentration increase result in a red shift of the Gold surface-plasmon resonance energy. The red shift due to the size effect is concluded as mainly originating from the sizedependent dielec. function of Gold core electrons and a semiquant. description of its size dependence is presented. The experimental result describing the concentration effect suggests a deviation from the conventional Maxwell-Garnett effective medium theory, which can be corrected by introducing the multipole effect based on the dipole-dipole interaction model.

\subsection{Electrochemistry of Ferrocene-modified monolayer-protected Gold Nanoclusters at Reduced Temperatures}

The electrochemical of ferrocene-modified monolayerprotected Gold nanocluster (Fc-MPC) solutions at reduced temperatures was studied. K. Kennedy, M. Grace, T. Deon from the Department of Chemistry, University of the South, Sewanee, USA, Journal of Undergraduate Chemistry Research 2004, 3(4), 145. Fc-MPC diffusion coefficients are established from macroelectrode cyclic voltammetry (CV) and shown to decrease as the solution temperature is lowered. Diffusion coeffs. were also detected for the ferrocenecarboxylic acid monomer (Fc- $\mathrm{COOH}$ ) at reduced temperatures with similar effects. The potential peak spacing $(\Delta \mathrm{Ep})$ for both species increases as the solution temperature is lowered. This effect is largely attributed to the increased solution resistance rather than the increased solvent viscosity at lower temps.

\section{$4 \quad$ Electrochemistry}

\subsection{Electrochemical Self-Assembly of Melanin Films on Gold}

An electrochemical method for self-assembling melanin films on the Gold(111) surface from melanin aggregates in alkaline media is reported. Electrochemical data combined with scanning tunneling microscopy (STM), at. force microscopy, and Auger electron spectroscopy show that the amount and structure of the deposited melanin film depend on the potential (E) applied to the electrochemical interface and deposition time. P. Diaz, Y. Gimeno, P. Carro, S. Gonzalez, P. Schilardi, G. Benitez, R. Salvarezza, A. Creus, A. Hernandez from Departamento de Quimica Fisica, Universidad de La Laguna, La Laguna, Tenerife,Spain Langmuir 2005, 21(13), 5924. Film formation takes place at a noticeable rate at $\mathrm{E}=-1.0 \mathrm{~V}$ (vs. SCE). High-resolution STM images at the early stages of growth show small particles, $5-8 \mathrm{~nm}$ in size and 0.3-0.4 nm in height, forming ordered arrays that follow closely the Cold(111) topography. The size of the melanin particles increases as the film thickness increases reaching $150 \mathrm{~nm}$ for deposits grown for $16 \mathrm{~h}$. The deposited films are electrochemical active, showing well-defined redox couples preceding the hydrogen evolution reaction. 


\subsection{Design and Fabrication of a Gold Electroplated Electromagnetic and Electrostatic Hybrid Mems Relay}

This paper reports on a Gold electroplated hybrid microrelay that combines the advantages offered by electromagnetic and electrostatic actuation mechanisms. S. Guan, K. Vollmers, A. Subramanian, B. Nelson, J. from Department of Mechanical Engineering, University of Minnesota, Minneapolis, MN, 55455, USA SO Journal of Applied Physics, 2005, 97(10, Pt. 3), 10R506/1. The microrelay design achieves signal by out-of-plane motion of a contact cross-bar fabricated on a diaphragm fixed at both ends to short the isolated input and output contacts. Major factors influencing the design approach include low contact resistance in the closed state, high signal isolation in the open state, latching upon loss of power, and fabrication robustness and cost. The MEMS device is surface micromachined with structures fabricated by electroplating Gold through photoresist masks. 95\% of the devices can be actuated and .apprxeq.50\% of them pass signal. The average contact resistance is .approximately $170 \mathrm{~m} \Omega$ and the system isolation is $70 \mathrm{~dB}$ at $20 \mathrm{MHz}$ when a Gold ground plane is added.

\subsection{Electrochemistry of Gold in Aqueous Sulfuric Acid Solutions under Neural Stimulation Conditions}

The electrochemical of Gold in $50 \mathrm{mM}$ aqueous $\mathrm{H}_{2} \mathrm{SO}_{4}$ solutions under conditions typical of neural stimulation (cathodic-first current pulsing at $50 \mathrm{~Hz}$ ) was studied in oxygen-free and oxygenated solutions D. Merrill, I. Stefan, D. Scherson, T. Mortimer from the Applied Neural Control Laboratory, Case Western Reserve University, Cleveland, USA, Journal of the Electrochemical Society 2005, 152(7), E212 . As the electrode was pulsed from $+0.75 \mathrm{~V}$ vs. a ref. hydrogen electrode using a $100 \mu \mathrm{s},-500 \mathrm{~mA} / \mathrm{cm}^{2}$ pulse in oxygen-free solutions, (i) the initial $10 \mu \mathrm{C} / \mathrm{cm}^{2}$ of injected charge was stored in the double layer, (ii) the next $20-30 \mu \mathrm{c} / \mathrm{cm}^{2}$ was accommodated by double-layer charging plus an undetd. process consuming $\approx 1.8 \mu \mathrm{C} / \mathrm{cm}^{2}$, and (iii) when the electrode potential reached $\approx-0.52 \mathrm{~V}, \mathrm{H}_{2} \mathrm{O}$ reduction consumed all further charge. In oxygen-satd. solution, the unrecoverable charge (assocd. with irreversible reactions) was not significantly different from the deaerated situation throughout the pulse period, but the electrode potential was less neg., consistent with oxygen reduction Oxygen reduction appears reversible during a $100 \mu$ s pulse, provided the electrode is clamped back to $+0.75 \mathrm{~V}$ immediately after the pulse. If an open-circuit period was introduced between the current pulse and application of the reversal potential, unrecoverable charge accumulated more rapidly in oxygensaturated solution, suggesting that processes occurring during the interpulse interval of neural stimulation may produce potentially damaging reaction products.

\section{$5 \quad$ Electronics and Sensors}

\subsection{Gold Finger Stacked Multichip Integrated Circuit Package for Mobile Phones}

The present invention provides an improved structure of gold fingers, which is to redesign a conventional gold finger on a packaging substrate into a gold finger set that contains a plurality of gold finger units. K. Wu, Patent No. US 2005156303 A1 Sept 2005. Between each single gold finger unit, there exists an elec. connection. Therefore, in the structure of stacked-chip packaging, each wire that is connected through wire bonding on the same gold finger of each layer chip can sep. perform wire bonding on different gold finger units of the same gold finger set. Due to the improvement on the gold finger structure, the present invention can prevent the adhesive on a chip from flowing along the wire bonding path of a layer chip and smearing the whole gold finger. Thus, other layer chips can be prevented from being unable to perform wire bonding.

\subsection{Semiconductor Device and its Manufacture for Preventing Abnormal Diffusion of Gold}

The claimed semiconductor device is equipped with a Pt layer and a Gold layer formed by electron beam deposition on a semiconductor layer to give an electrode containing the Pt layer or the Gold layer forming Shottkey junction or ohmic junction, where the electrode has a thin layer at end containing only the Pt layer or the Gold layer. M. Shiota from the Sharp Corp., Japan, Patent No. JP 2005191372, A2 Sept 2005. The claimed semiconductor device, equipped with a Pt layer and a Gold layer, where one of which gives an electrode for forming Shottkey junction or ohmic junction, is manufd. by steps of (1) electron beam deposition of the electrode material on the semiconductor substrate, (2) removing layers of the Pt layer or the Gold layer not forming the Shottkey junction or ohmic junction, and then (3) forming an electrode by lift-off method.

\subsection{Polyaniline Nanofiber/Gold Nanoparticle Nonvolatile Memory}

A nonvolatile plastic digital memory device based on nanofibers of the conjugated polymer polyaniline decorated with gold nanoparticles is reported. R. Tseng, J. Huang, J. Ouyang, R. Kaner, Y. Yang from the Department of Materials Science and Engineering, University of California, Los Angeles, USA, Nano Letters 2005, 5(6), 1077. The device has a simple structure consisting of the plastic composite film sandwiched between two electrodes. An external bias was used to program the ON and OFF states of the device that are separated by a 3-orders-of-magnitude difference in cond. ON-OFF switching times of $<25$ ns are observed by elec. pulse measurements. The devices possess prolonged retention times of several days after they were programmed. Writeread-erase cycles are also demonstrated. The switching mechanism is attributed to an elec.-field-induced charge 
transfer from the polyaniline nanofibers to the gold nanoparticles. The active polymer layer is created by growing nanometer size gold particles within 30-nm-diameter polyaniline nanofibers using a redox reaction with chloroauric acid. This device combines two exciting research areasnanoparticles and conducting polymers - to form a novel materials system with unique functionality.

\subsection{A Novel Method for Enabling the Thermosonic Wire Bonding of Gold Wire onto Chips with Copper Interconnects}

The wire bonding of chips with copper interconnects is a challenging issue. Y. Jeng, C. Wang, S. Chiu, C. Cheng from the Department of Mechanical Engineering, National Chung Cheng University, Taiwan, Electrochemical and Solid-State Letters 2004, 7(12), G335. The present study develops a method in which the substrates are stored in a temperature and humidity controlled environment such that copper oxide films of appropriate thickness are formed on the substrate prior to the gold-copper thermosonic bonding process. It is shown that this film can be removed during the bonding process, hence rendering the wire bonding process feasible without the requirement for an inert gas environment or the use of metallic cap layers.

\section{$6 \quad$ Medical and Dental}

\subsection{Preparation of Functionalized and Gold- Coated Cobalt Nanocrystals for Biomedical Applications}

Y. Bao, K. Krishnan from the Department of Materials Science and Engineering, University of Washington, Seattle, USA, Journal of Magnetism and Magnetic Materials 2005, 293(1), 15 present the synthesis, structural and magnetic characterization of cobalt-based nanocrystals with controlled size (5-25 nm; .+-.1 nm) and tailored morphologies (spheres, disks with specific aspect ratio of $5 \times 20 \mathrm{~nm}$ and core-shell structures). The reproducible synthesis route for Gold-coated, high moment, cobalt nanocrystals presented here opens up possibilities for a number of biomedical applications and surface functionalities.

\section{$7 \quad$ Metallurgy, Materials and Coatings}

\subsection{Magnetic and Optical Tunable Microspheres with a Magnetite/Gold Nanoparticle Shell}

M. Spasova, V. Salgueirino-Maceira, A. Schlachter, M. Hilgendorff, M. Giersig, L. Liz-Marzan, M. Farle, from the Fachbereich Physik, Universitaet Duisburg-Essen, Duisburg, Germany, Journal of Materials Chemistry 2005, 15(21), 2095. Multifunctional core-shell microspheres consisting of a polystyrene $640 \mathrm{~nm}$ diam. core covered with a selectable no. of layers of $\mathrm{Fe}_{3} \mathrm{O}_{4}(12 \mathrm{~nm})$ and $\mathrm{SiO}_{2}$-coated Gold $(15 \mathrm{~nm})$ nanoparticles were fabricated using the layer-by-layer technique in aqueous solution. By varying the shell thickness and layer composition the magnetic and optical properties and the diam. of the colloids can be controlled independently. Optical spectra of the core-shell colloidal microspheres show a well-resolved Gold surface plasmon peak in the visible which red shifts with the no. of adsorbed Gold nanoparticle layers. The magnetic moment per sphere increases nearly linearly with the number of adsorbed magnetite nanoparticle layers, and the spheres can be assembled into chains of .Itoreq. $1 \mathrm{~mm}$ length by deposition in a magnetic field.

\subsection{Role of Gold in High Temperature Oxidation of Titanium Alloy}

Oxidation of $\mathrm{Ti}$ and its alloys at a high temperature for a short time was carried out with Gold and Pt predeposition. Z. Zhang, H. Dong, T. Bell from the Surface Engineering Research Institute of CMES, Peoples Republic of China, Surface Engineering 2005, 21(1), 76. Preliminary results show Gold can improve the quality of the oxide and the bond strength of the metal/oxide. Gold predeposition leads to a thicker oxide layer and more absorption of O. Further investigations show that the existence of the thin Gold layer is beneficial for the outward diffusion of Ti during oxidation at $800^{\circ}$ and $850^{\circ}$.

\subsection{Gold Seed-Assisted Synthesis of Silver Nanomaterials under Microwave Heating}

F. Liu, P. Huang, T. Chu, F. Ko from the National Nano Device Laboratories, Hsinchu, Taiwan, Materials Letters 2005, 59(8-9), 940 report a low-temperature process for prepg. templateless and polymerless $\mathrm{Ag}$ nanomaterials in the presence of Gold seeds under microwave (MW) heating. In addition to spherical nanoparticles, Ag nanorods are also produced. Key aspect for the production of nanorods is the addition of adequate vol. of Gold seeds. The combination of MW heating synthesis method in the presence of Gold seeds is a good approach to prepared templateless and polymerless Ag nanorods.

\section{$8 \quad$ Nanotechnology}

\subsection{Phase Properties of Carbon-Supported Gold-Platinum Nanoparticles with Different Bimetallic Compositions}

The ability to control the composition and phase properties of bimetallic nanoparticles is crit. in exploring catalytic properties. In this paper J. Luo, M. Maye, V. Petkov, N. Kariuki, L. Wang, P. Njoki, D. Mott, Y. Lin, C.J. Zhong from the Department of Chemistry, State University of New York at Binghamton, Binghamton, USA, Chemistry of Materials 2005, 17(12), 3086 present results from a study aimed at detg. those properties for carbon-supported gold-platinum (AuPt) 
catalysts with different bimetallic compositions. The bimetallic nanoparticle catalysts are prepared by a two-phase synthesis protocol employing org. monolayer encapsulation on bimetallic AuPt cores $(\approx 2 \mathrm{~nm})$. The size-controlled nanoparticles are assembled on carbon black support materials with controllable dispersion and metal loading and are further treated by calcination under controlled temperature and atm. The core composition of the bimetallic nanoparticles is detected by d.c. plasma-at. emission spectroscopy. Structural characterization is carried out by X-ray diffraction. The bimetallic nanoparticles were shown to display alloy properties, which is in sharp contrast to the bimetallic miscibility gap known for the bulk counterpart of the bimetallic metals. This finding demonstrates the difference of the phys. and chem. properties for nanoscale materials from the bulk crystalline state, revealing important details of the phase properties of the bimetallic nanoparticle catalysts and new information for the correlation between the composition and the phase properties at the nanoscale. Implications of their findings to the design and manipulation of the bimetallic nanoparticles for catalytic applications are also discussed.

\subsection{Facile One-Pot Synthesis of Gold Nanoparticles Stabilized with Bifunctional Amino/Siloxy Ligands}

A method for the direct one-pot synthesis of amine-stabilized gold nanoparticles using 3-(trimethoxysilylpropyl)diethylenetriamine (TMSP dien) is described. H. Zhu, Z. Pan, E. Hagaman, C. Liang, S. Overbury, S. Dai, Sheng from the Chemical Sciences Division, Oak Ridge National Laboratory, Oak Ridge, USA, Journal of Colloid and Interface Science 2005, 287(1), 360. The amine groups of this bifunctional mol. act as a stabilizer for gold nanoparticles as they form by reduction of $\mathrm{HAuCl}_{4}$. Highly stable gold nanoparticles with sizes tunable between 8 and $20 \mathrm{~nm}$ can be readily obtained. This method is quite simple to implement and environmentally benign as there is no need to add an external reducing reagent. The incorporated siloxy functionality was subsequently used to form a silica shell around the gold particle.

\subsection{Fine-Tuning the Shape of Gold Nanorods}

Gold nanorods prepared by a seed-mediated growth approach using $4 \mathrm{~nm}$ gold nanospheres as the seeds and subsequent reduction of metal salt with a weak reducing agent (ascorbic acid) in the presence of a directing surfactant to produce nanorods. L. Gou, C. Murphy from the Department of Chemistry and Biochemistry, University of South Carolina, Columbia, USA, Chemistry of Materials 2005, 17(14), 3668. If insufficient ascorbic acid is added in the growth step, then metal salt remains. Addnl. input of ascorbic acid preferentially deposits more metal at the ends of the nanorods, to yield "dogbone"-like structures. Surprisingly, heat treatment of the unpurified gold nanorods (prepared with an insufficient amt. of ascorbic acid) yielded fatter gold nanorods; the oxidation product of ascorbic acid appears to act as a reductant at higher temperature These modified shapes of the gold nanorods directly influence their optical properties.

\subsection{Sono- and Photochemical Routes for the Formation of Highly Dispersed Gold Nanoclusters in Mesoporous Titania Films}

J. Yu, X. Wang, L. Wu, W. Ho, L. Zhang, G. Zhou from the Department of Chemistry and Environmental Science Programme, The Chinese University of Hong Kong, Hong Kong, Peoples Republic of China, Advanced Functional Materials 2004, 14(12), 1178. A sono- and photochem. approach was developed to incorporate highly dispersed Gold nanoclusters into mesoporous $\mathrm{TiO}_{2}$ films. The first step involves the sonication of a $\mathrm{TiO}_{2}$ film immersed in a Gold chloride solution This effectively removes the air trapped in the porous film matrix and drives the Gold chloride into the pore channels, leading to a homogeneous adsorption of ionic Gold in the $\mathrm{TiO}_{2}$ mesoporous matrix. The second step takes advantage of the photocatalytic property of $\mathrm{TiO}_{2}$ to reduce the adsorbed Gold ions to AuO. As the Gold nanoclusters thus produced are stabilized by the $\mathrm{TiO}_{2}$ mesonetwork, no org. capping mols. are required. Highly dispersed $\mathrm{Au} / \mathrm{TiO}_{2}$ nanoheterojunction arrays can be obtained using this interesting approach.

\subsection{One-Step Preparation of Highly Concentrated Well-Stable Gold Colloids by Direct Mix of Polyelectrolyte and $\mathrm{Haucl}_{4}$ Aqueous Solutions at Room Temperature}

X. Sun, S. Dong, E. Wang from the Graduate School of the Chinese Academy of Sciences, Changchun Changchun, Peoples Republic of China, Journal of Colloid and Interface Science 2005, 288(1), 301

Highly concentrated, well-stable Gold colloids can be prepared directly from an amine-bearing polyelectrolyte$\mathrm{HAuCl}_{4}$ aqueous solution at room temperature Increasing molar ratio of polyelectrolyte to Gold leads to increasing particle size. UV-visible spectra, TEM, and $\mathrm{x}$-ray photoelectron spectra (XPS) were used to characterize the products thus formed.

\subsection{Coordination-Based Gold Nanoparticle Layers}

Gold nanoparticle (NP) mono- and multilayers were constructed on gold surfaces using coordination chemistry, M. Wanunu, R. Popovitz-Biro, H. Cohen, A. Vaskevich, I. Rubinstein from the Departments of Materials and Interfaces and Chemical Research Support, Weizmann Institute of Science, Rehovot, Israel, Journal of the American Chemical Society 2005, 127(25), 9207. Hydrophilic Au NPs (6.4 nm av. core diam.), capped with a monolayer of 6-mercaptohexanol, were modified by partial substitution of bishydroxamic acid disulfide ligand mols. into their capping layer. A monolayer of the ligand-modified Au NPs was 
assembled via coordination with $\mathrm{Zr}^{4+}$ ions onto a semitransparent Gold substrate (15 nm Au, evapd. on silanized glass and annealed) precoated with a selfassembled monolayer of the bishydroxamate disulfide ligand. Layer-by-layer construction of NP multilayers was achieved by alternate binding of $\mathrm{Zr}^{4+}$ ions and ligand-modified NPs onto the first NP layer. Characterization by at. force microscopy (AFM), ellipsometry, wettability, transmission UV-vis spectroscopy, and cross-sectional transmission electron microscopy showed regular growth of NP layers, with a similar NP d. in successive layers and gradually increased roughness. The use of coordination chem. enables convenient step-bystep assembly of different ligand-possessing components to obtain elaborate structures. This is demonstrated by introducing nanometer-scale vertical spacing between a NP layer and the gold surface, using a coordination-based org. multilayer. Elec. characterization of the NP films was carried out using conductive AFM, emphasizing the barrier properties of the org. spacer multilayer. The results exhibit the potential of coordination self-assembly in achieving highly controlled composite nanostructures comprising mols., NPs, and other ligand-derivatized components.

\subsection{Preparation of Gold Nanoparticles by Heat Treatment of Sputter Deposited Gold on Boron-Doped Diamond Film Electrode}

A novel two-step method was developed to prepared gold nanoparticles on boron-doped diamond (BDD) electrode. B. El Roustom, G. Foti, C. Comninellis from the Institute of Chemical Sciences and Engineering, Ecole Polytechnique Federale de Lausanne (EPFL), Lausanne, Switzerland, Electrochemistry Communications 2005, 7(4), 398. It consisted of sputter deposition of max. 15 equiv monolayers of gold, followed by a heat treatment at $400-600^{\circ}$ in air. The as-sputtered gold deposits were highly dispersed (estd. particle size of 2-7 nm) but also unstable, a continuous potential cycling in the stability region of sulfuric acid leading to complete loss of the deposited gold. Subsequent heat treatment of as-sputtered deposits resulted in formation of a stable population of nanosized gold particles characterized by SEM and cyclic voltammetry. Gold nanoparticles with an av. size of 15-35 nm could be prepared by this method on polycryst. BDD film electrode, the particle size being dependent of the deposited amt. The invariant voltammetric response of the heat-treated gold deposits confirmed their applicability for electrocatalysis.

\subsection{Size Controlled Gold Nanoparticle Formation by Avena Sativa Biomass: Use of Plants in Nanobiotechnology}

Oat (Avena sativa) biomass was studied as an alternative to recover $A u(I I)$ ions from aqueous solutions and for its capacity to reduce $\mathrm{Au}(\mathrm{III})$ to $\mathrm{Au}(0)$ to form Gold nanoparticles. V. Armendariz, I. Herrera, J. Peralta-Videa, M. Jose-Yacaman, H. Troiani, P. Santiago, J. Gardea-Torresdey from the Department of Chemistry, University of Texas at
El Paso, USA, Journal of Nanoparticle Research 2004, 6(4), 377. To study the binding trend of $\mathrm{Au}(\mathrm{III})$ to oat and the possible formation of Gold nanoparticles, the biomass and a solution of $\mathrm{Au}$ (III) were reacted for $1 \mathrm{~h}$ at $\mathrm{pH}$ 2-6. $\mathrm{Au}$ (III) ions were bound to the oat biomass in a $\mathrm{pH}$-dependent manner, with the highest adsorption of $\approx 80 \%$ at $\mathrm{pH} 3$. High-resolution TEM studies showed that the oat biomass reacted with $\mathrm{Au}(\mathrm{III})$ ions to form Gold nanoparticles with a fcc. tetrahedral, decagonal, hexagonal, icosahedral multi-twinned, irregular, and rod shapes. The $\mathrm{pH}$ of the reaction influenced the nanoparticle size. The smaller nanoparticles and higher occurrence of these were observed at $\mathrm{pH} 3$ and 4, whereas the larger nanoparticles were observed at $\mathrm{pH} 2$.

\subsection{Preparation of Nanosized Gold Particles in a Biopolymer Using UV Photoactivation}

Gold nanoparticles were prepared by UV photoactivation in the presence of a biopolymer, Na alginate. The particles are characterized by UV-visible spectra and TEM studies. A. Pal, K. Esumi, T. Pal from the Department of Civil Engineering, Indian Institute of Technology, Kharagpur, India, Journal of Colloid and Interface Science 2005, 288(2), 396. Both particle size and the UV-visible absorption peak are dependent on the $\mathrm{Na}$ alginate concentration The effects of various other parameters such as change of light source, cell material of the reaction chamber, heating effect, irradn. time, and $\mathrm{HAuCl}_{4}$ concentration are studied. The particles are spherical and in situ stabilized by the biopolymer. The method is very simple and reproducible.

\section{$9 \quad$ Refining}

\subsection{Recovery of Gold from Gold Slag by Wood Shaving Fly Ash}

Wood shaving fly ash was used as an alternative adsorbent for gold preconcentration from gold slag. A. Aworn, P. Thiravetyan, W. Nakbanpote from the School of Energy and Materials, King Mongkut's University of Technology Thonburi, Bangkok, Thailand, Journal of Colloid and Interface Science 2005, 287(2), 394. The max. gold adsorption capacity of wood shaving fly ash washed with tap water (WSFW) at $20,30,40$, and $60^{\circ} \mathrm{C}$ was $8.68,7.79,7.44$, and 7.25 $\mathrm{mgAu} /$ gadsorbent, respectively, while of activated carbon it was 76.78, 60.95, 56.13, and 51.90 mgAu/gadsorbent, respectively Deionized water at $100^{\circ}$ could elute gold adsorbed onto WSFW to $71 \%$. The effect of the increasing temperature of water, 30,60 , and $100^{\circ}$ implied that the adsorption mechanism was mainly phys. adsorption. The negative values of enthalpy change $(\Delta H)$ and free energy change $(\Delta \mathrm{G})$ indicated an exothermic and spontaneous process, respectively The pos. values of entropy change $(\Delta S)$ indicated increasing disorder of the system. The advantages of wood shaving fly ash are the purifn. of gold and the easier recycling of gold from the gold-adsorbed adsorbent. 


\subsection{Solvent Extraction of $\mathrm{Au}(\mathrm{l})$ from Gold-Thiourea Leaching Solution by Alkyl Benzothiazole Sulfoxides}

The capability of extn. $\mathrm{Au}(I)$ with Bu benzothiazole sulfoxide (NBBSO) from gold-thiourea leaching solution was investigated. Y. Li, G. Gu, L. Li, Z. Xu from the Department of Applied Chemistry, South China University of Technology, Guangzhou, Peoples Republic of China, Guijinshu 2004, 25(4), 1. The $95 \%$ extn. rate was obtained with $2.3 \mathrm{~mol} / \mathrm{L}$ NBBSO in $0.200 \mathrm{mg} / \mathrm{mL} \mathrm{Au}(\mathrm{l}), 0.08 \mathrm{~mol} / \mathrm{L}[\mathrm{H}+]$ solution This rapid reaction reached equil. in 5 min. 99.2\% stripping of $\mathrm{Au}(\mathrm{I})$ was achieved with $12.5 \% \mathrm{Na}_{2} \mathrm{SO}_{3}$. and the structural effects of alkyl(benzyl) benzothiazole sulfoxides on the extn. of $\mathrm{Au}(\mathrm{l})$-thiourea leaching solution were reported. The extn. behavior of these sulfoxides may be affected by charge $d$. of coordination group and steric substituents effects.

\subsection{Recovery of Gold Fines from Geological Exploration Samples under Field Conditions}

Transportable mechanical application for crushing and milling of geol. rock samples was developed for beneficiation and improved recovery of Gold fines and flakes. V. Man'kov, Razvedka i Okhrana Nedr 2004, (8-9), 47. The application has nominal recovery of $\approx 99 \%$ at the Gold particle size of $0.4 \mu \mathrm{m}$, decreasing to $95 \%$ at $0.1 \mu \mathrm{m}$. The application and process are suitable for processing of geol. samples containing $\approx 100 \mathrm{mg} \mathrm{Au} / \mathrm{m}^{3}$.

\subsection{Increasing Gold Recovery in Heap Leaching Practice}

To increase gold recovery in Gaolong heap leaching plant, the problems with the initial process were studied and efforts were made in improving the process. S. Li from the Gaolong gold Mining Ltd. of Tianlin County of Guangxi Zhuangzu Autonomous Region, Peoples Republic of China, Huangjin 2004, 25(6), 45. The grain of ore particles should be under $50 \mathrm{~mm}$. The former process was changed into layered heaping, layered spraying, and heaping and spraying were at the same time. PH of pre-leaching gold solution before spraying was regulated to approx. 10 , and the mass fraction of $\mathrm{CN}$ - in leaching solution should be at 0.01\%-0.03\%. Through one-year production with the improved process, gold recovery has been lifted from $60 \%$ up to $80 \%$.

\subsection{Improvements in the Cyanide Reduction Process at Chatree Gold Mine}

The Chatree Gold Mine is currently operating in Thailand, and is restricted to the total cyanide discharge of .Itoreq.20 ppm in the plant discharge slurry prior to release into the tailings storage facility. W. Saranrittichai, K. Niyomjinda, B. Etschmann from the Chatree Gold Mine Metallurgical Department, Akara Mining, Phichit, Thailand, Publications of the Australasian Institute of Mining and Metallurgy 2004, 4/2004), 413. The compliance with this limit is based on the modified Inco $\mathrm{SO}_{2}$-Air cyanide destruction system. The assocd. improvements were focussed on decreasing the formation of cyanide and in the ore-leaching circuit, and improved removal of the ferrocyanide species from solution during the cyanide reduction stage.

\subsection{Method of Extraction of Gold and Silver from Polymetallic Raw Material}

The invention by T. Avaeva, S. Belov, G. Seredina from the Moskovskaya Gosudarstvennaya Akademiya Tonkoi Khimicheskoi Tekhnologii im. M. V. Lomonosova, Patent No RU 2258768 , C1 August 2005, is pertaining to the field of hydrometallurgy of noble metals, in particular, to the methods of extn. of gold and silver from various kinds of polymetallic raw materials, composition of which may include copper, nickel, tin, lead, noncorrosive steel and other metals. The tech. result ensures a selective extn. of gold and silver from the current-conducting materials ensuring the high speeds of dissolution of precious metals. The method provides for a treatment of a material placed in the anodic chamber of an electrolyzer and acting as an anode with an acid solution of thiourea (25-100 g/l, pH = 0.5-2.0), passing of a d.c. flow and realization of an electrolysis at the temperature of $20-35^{\circ} \mathrm{C}$ in a controlled potential mode at potential of the anode $=+0.380$.div. $+0.420 \mathrm{~V}$ in respect to a normal hydrogen electrode. The speed of the gold dissolution reaches $0.43 \mathrm{mg} / \mathrm{cm}^{2}$ per a minute, silver -0.37 $\mathrm{mg} / \mathrm{cm}^{2}$ per a minute. The degree of extn. of gold makes 99.9\%, silver $-99.6 \%$.

\subsection{Optimization of Pulp Density and Particle Size in the Biooxidation of a Pyritic Gold Concentrate by Sulfolobus Metallicus}

Although increasing pulp $d$. and decreasing particle size have positive effects in the volumetric rate of biooxidation of refractory gold concs., a variety of phenomena such as mech. damage, metabolic stress and inhibition can limit this effect. F. Acevedo, J. Gentina, P. Valencia from the School of Biochemical Engineering, Catholic University of Valparaiso, Chile, World Journal of Microbiology \& Biotechnology 2004, 20(8), 865. The objective was to det. the pulp d. and particle size values that maximize the volumetric solubilization rate of iron from a pyritic gold conc. The leaching was carried out in agitated flasks with the thermophilic archaeon Sulfolobus metallicus. The conc. contained $66.7 \%$ pyrite, and the const. operation conditions were 220 revolutions/min, $68^{\circ}$ and initial pH 2.0. Pulp densities were 2.5, 5, 10 and $15 \%$ wt./vol., and the size fractions were 150-106, 106-75, 75-38 and $<38 \mu \mathrm{m}$. Total solubilized iron concns. were in the range of $8-25 \mathrm{~g} / \mathrm{L}$. In the 2.5 and $5 \%$ pulp $d$. runs, iron extns. were in the range 80-100\%. A complete experimental design of 16 runs allowed the building of response surfaces from which the optimum conditions that maximize the rate of iron solubilization were detd. These conditions were $7.8 \%$ pulp d. and particle size $35 \mu \mathrm{m}$. 


\subsection{Minataur: the Mintek Alternative Technology to Gold Refining}

Mintek developed the Minataur leach/solvent extn./precipitation technology as a cost-effective, easily operated alternative to conventional gold refining processes. S. Scott, K. Matchett from Mintek, Randburg, South Africa, Journal of the South African Institute of Mining \& Metallurgy 2004, 104(6), 339. The technolology was first commercialized at the Harmony Gold Refinery in 1997 to produce $99.99 \%$ gold product and has subsequently led to development of various value-addition steps including the casting of various investment bars, gold wire drawing and gold potassium cyanide powder production In addition to refining gold to high purity, if an appreciable amt. of silver is present in the feed it is also refined to $99.99 \%$ purity. The major advantage that the Minataur technol. has over other gold refining processes is the relatively low gold lock-up and reduced operating costs. The operating cost, as at Apr. 2001 for the expanded Harmony refinery 24-ton/yr gold prodn. was approx. half that of conventional smelting and toll refining. The extractant employed for solvent extn. is relatively cheap and highly selective for gold to make the technol. attractive in situations where significant quantities of platinum group metals or base metals are present.

\section{General}

\subsection{Research on the Traditional Metal Decoration using Gold Foil (I Report)}

Decorative items comprising Ag substrate and Gold foil layer had been made in old times. However, this technique is not used in jewelry industries now. K. Morimoto, F. Suzuki, K. Kobayasi from Yamanashi Prefectural Industrial Technology Center, Japan Yamanashi-ken Kogyo Gijutsu Senta Kenkyu Hokoku 2003, 17, 118. Experiments have been carried out in this study to investigate the effects of foil thickness, temperature, Ag substrate on the quality of pressure bonding. Several samples were fabricated using the technique.

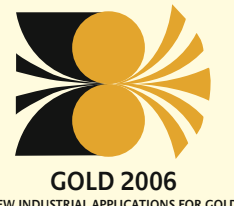

NEW INDUSTRIAL APPLICATIONS FOR GOLD

\title{
GOLD 2006 NEW INDUSTRIAL APPLICATIONS OF GOLD
}

\begin{abstract}
The 4th International Conference on Gold Science, Technology and its Applications will take place on
\end{abstract} 3rd-6th September, 2006, at the University of Limerick, Limerick, Ireland.

See website for full information.

Call for papers: Abstracts are invited for papers and posters by 16th January 2006.

Full details on conference website at: www.gold2006.org 